\title{
Synthesis of New 1,2,3-triazole-1,4-naphthoquinones
}

\author{
Wilson S. Nascimento ${ }^{1 *}$, Celso Amorim Camara, ${ }^{2}$ Ronaldo N. de Oliveira ${ }^{2}$ \\ ${ }^{1}$ Departamento de Química Fundamental, CCEN, UFPE, 50670-901, Recife-PE, Brasil \\ ${ }^{2}$ Departamento de Química, UFRPE, 52171-900, Recife, PE, Brasil \\ *e-mail:wilsonnascimentolq@hotmail.com
}

Keywords: triazole, cycloaddition, naphthoquinone

\section{INTRODUCTION}

The development of new molecular libraries for biological screening has gained impact among synthetic chemists. The $N$-heterocycle, namely 1,2,3-triazole, have received considerable attention in the search for new drugs in pharmacology. Hence, many efforts have been made in the optimization of their preparation methods. One example is the 1,3-dipolar cycloaddition reaction between an azide and a terminal alkyne to obtain this triazole, using a copper-based catalyst. ${ }^{1}$ Substituted naphthoquinones are reported to display important biological activity. ${ }^{2}$ For this reason, we turned our attention to the investigation of $\mathrm{N}$-triazole linked with 1,4-naphthaquinone, in the course of a project involving the synthesis and biological evaluation of a series of new heterocyclic derivatives.

\section{RESULTS AND DISCUSSION}

As a model to the cycloaddition reaction towards 1,2,3-triazole-linked 1,4-naphthoquinone, we choose to examine the reaction of 2-azido-1,4naphthoquinone (1) with phenylacetylene (2a), using a copper(I)-based catalyst. We examined some variables from our template reaction using $10 \mathrm{~mol} \%$ loading of $\mathrm{Cul}$ as $\mathrm{Cu}^{+}$source. Firstly, guided by our recent results, ${ }^{3}$ we employing the solvent dichloromethane (DCM) at $30^{\circ} \mathrm{C}$ without base or ligand additives, but the reaction did not proceed. Fortunately, substitution of $\mathrm{DCM}$ by $\mathrm{CH}_{3} \mathrm{CN}$ resulted in compound (3a) in good yields $80 \%$, after a reaction time of $24 \mathrm{~h}$. We decided to apply this protocol and we obtain 1,2,3-triazole-1,4naftoquinones (3a-e) in good yields $73-90 \%$ (Scheme 1).
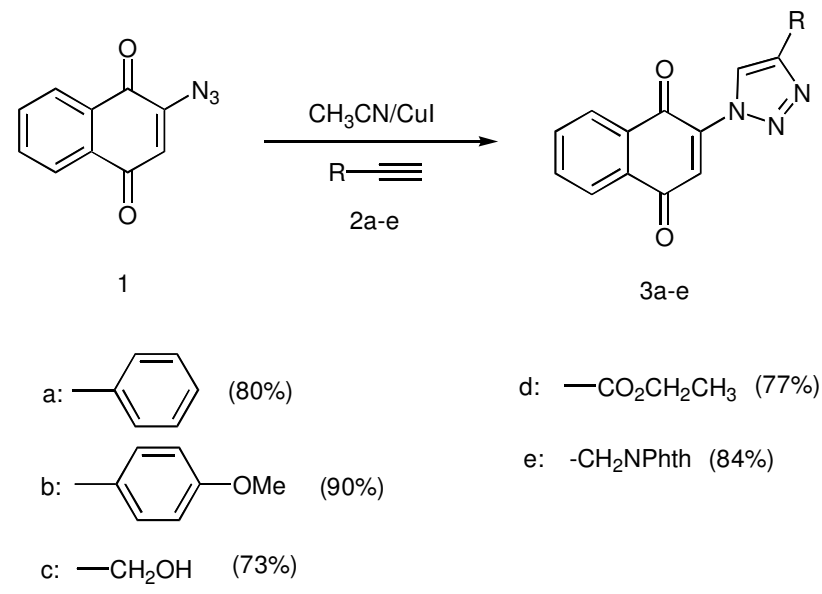

3a-e

Scheme 1. Synthesis of new 1,2,3-triazole-1,4naphthoquinones (3a-e)

\section{CONCLUSION}

We have shown that the reaction of 2-azide-1,4naphthoquinone (1) with a variety of substituted acetylenes (2a-e) does occur in $\mathrm{CH}_{3} \mathrm{CN}$ at room temperature, using $\mathrm{Cul}$ as a catalyst. This convenient synthesis using a click-chemistry protocol afforded 1,2,3-triazole-1,4-naphthoquinones (3a-e) in good yields (73-90\%).

\section{ACKNOWLEDGEMENTS}

The authors are grateful to FACEPE for financial support and for providing a fellowship to one of us (W.S.N.). Our thanks are also due to Analytical Centers CENAPESQ-UFRPE and DQF-UFPE.

\section{REFERENCES}

${ }^{1}$ Meldal, M.; Tornøe, C. W. Chem. Rev. 2008, 108, 2952.

${ }^{2}$ Cunha, A. S.; Lima, E. L. S.; Pinto, A. C.; Esteves-Souza, A.; Echevarria, A.; Camara, C. A.;Vargas, M. D.; Torres, S. J. J. Braz. Chem. Soc. 2006, 17, 439.

${ }^{3}$ Barbosa, F.C.G.; de Oliveira, R.N. J. Braz. Chem. Soc. 2011, 22, 592. 